\title{
Some Biological Effects of Volatile Metabolites from Cultures of Saccharomyces cerevisiae Meyen ex Hansen
}

\author{
By A. T. GLEN AND S. A. HUTCHINSON \\ Joint Mycological Laboratories, Departments of Botany and of \\ Chemistry, The University, Glasgow, W.2.
}

(Accepted for publication 2I August 1968)

\begin{abstract}
SUMMARY
The gas mixtures from cultures of Saccharomyces cerevisiae inhibited growth and sporulation of Aspergillus niger and germination of seeds of Lepidium sativum. In test conditions seven volatile organic metabolites in the culture gases were identified by gas-liquid chromatography (GLC) as acetaldehyde, ethyl acetate, ethanol, $n$-propanol, isobutanol, and a mixture of isopentanols (I part 2-methyl-butan-I-Ol to 2 parts 3-methyl-butan-I-ol); changes in the concentrations of $\mathrm{CO}_{2}$ and $\mathrm{O}_{2}$ were also measured. Pure samples of each of these components were tested at the concentrations found in the culture gases in order to identify the inhibitory substances.

Inhibition of growth of Aspergillus niger could be produced by these culture gas concentrations of acetaldehyde and of ethanol. Inhibition by this concentration of $\mathrm{CO}_{2}$ was just significant in these tests. The effect on sporulation could be produced by the $\mathrm{CO}_{2}$, but not by these concentrations of any of the other identified components.

The effect of Lepidium sativum seed germination could be produced by these culture gas concentrations of ethanol and of 3-methyl-butan-I-ol to a lesser extent. Slight effects were also observed with a lowered $\mathrm{O}_{2}$ concentration and with a raised $\mathrm{CO}_{2}$ concentration but not with the other constituents.
\end{abstract}

\section{INTRODUCTION}

Dick \& Hutchinson (1966) reported that gases from cultures of Saccharomyces cerevisiae inhibited the growth and/or sporulation of several species of fungi, and Hutchinson (1967) reported that they inhibited spore germination and prothallial growth of Pteridium aquilinum. Lösel (1964) summarized previous work on the biological effects of volatile fungal products. Robinson \& Park (1966) and Robinson, Park \& Garrett (1968) identified acetaldehyde as one of the volatile products from cultures of Fusarium oxysporum and of Rhizopus stolonifer which can inhibit fungal spore germination; they also identified ethanol amongst the nine otherwise unidentified peaks which they saw in gas-liquid chromatograms of samples from these cultures, but they found that it did not inhibit spore germination in their tests. They state that none of the volatile germination inhibitors affected the growth of hyphae "to any marked degree' in their tests. Norrman (1968) examined the effects of vapours from aqueous solutions of a variety of organic compounds on the morphology of Pestalotia rhododendri. He found that spore production was stimulated by many compounds in the order of activity alcohols $>$ esters $>$ acids $>$ aldehydes $>$ ketones, and that high vapour concentrations of many of these substances often inhibited linear growth of 
the colony. We now report on the analysis of the gas mixtures produced by $S$. cerevisiae in defined conditions, and on the identification of the active components.

\section{METHODS}

\section{Analysis of the gases above yeast cultures}

Cultures of Saccharomyces cerevisiae Meyen ex Hansen (Glasgow University Collection I, referred to as 'yeast' throughout this article) were prepared by spreading a suspension of cells over the surface of $200 \mathrm{ml}$. of $5 \%$ malt agar $(50 \mathrm{~g}$. Oxoid malt extract $+20 \mathrm{~g}$. Oxoid agar in $\mathrm{I} 1$. of deionized water) in each of a series of $\mathrm{I} 1$. Roux bottles. The cultures were then incubated at $30^{\circ}$ in the dark for $48 \mathrm{~h}$. Five ml, I ml. and $0.5 \mathrm{ml}$. were then withdrawn by syringe through the cotton-wool plug for analysis of organic volatiles, $\mathrm{O}_{2}$ and $\mathrm{CO}_{2}$ respectively. Similar samples were also taken from the assemblies used to examine the biological effects of these gases (described below).

The samples were analysed in an Aerograph Model 204 gas-liquid chromatograph, using a flame ionisation detector for organic volatiles and a micro cross-section detector for $\mathrm{CO}_{2}$ and $\mathrm{O}_{2}$, under the operating conditions shown in Table I.

Table I. Operating conditions used for chromatographic analysis of yeast culture gases

\begin{tabular}{|c|c|c|c|c|c|c|}
\hline Column & Dimensions & Material & Packing & $\begin{array}{l}\text { Temp. } \\
\left({ }^{\circ} \mathrm{C}\right)\end{array}$ & $\begin{array}{l}\text { Carrier gas } \\
\text { and flow } \\
\text { rate } \\
\text { (ml./min.) }\end{array}$ & $\begin{array}{c}\text { Hydro- } \\
\text { gen flow } \\
\text { rate } \\
\text { (ml./ } \\
\text { min.) }\end{array}$ \\
\hline A & Io $\mathrm{ft} \times \frac{1}{4}$ in & S.S.* & $\begin{array}{l}\text { Molecular sieve } \\
\text { 5A 30/60 mesh }\end{array}$ & 65 & $\mathrm{He} 22$ & - \\
\hline B & 14 in $\times \frac{1}{4}$ in & Copper & $\begin{array}{l}\text { Davison o8 grade } \\
\text { silica gel } 30 / 60 \text { mesh }\end{array}$ & 50 & $\mathrm{He} 30$ & - \\
\hline C & $5 \mathrm{ft} \times \frac{1}{8}$ in & S.S. & $20 \%$ Carbowax 1500 & 65 & $\mathrm{~N}_{2} 25$ & 25 \\
\hline D & $6 \mathrm{ft} \times \frac{1}{8}$ in & S.S. & $15 \%$ Dinonyl phthalate & 60 & $\mathrm{~N}_{2} 30$ & 30 \\
\hline $\mathbf{E}$ & $6 \mathrm{ft} \times \frac{1}{8}$ in & S.S. & $\begin{array}{l}20 \% \text { I,2,3-Tris- } \\
\text { (2-cyanoethoxy) } \\
\text { propane }\end{array}$ & 50 & $\mathrm{~N}_{2} 30$ & 25 \\
\hline$F$ & $6 \mathrm{ft} \times \frac{1}{4}$ in & Copper & $25 \%$ glycerol & 60 & $\mathrm{~N}_{2} 30$ & 25 \\
\hline
\end{tabular}

The constituents of the culture gases were identified by comparison with retention times of authentic pure samples and by syringe reactions (Hoff \& Feit, 1964). The concentration of each identified metabolite was estimated by comparing the height of the peaks in representative samples with those of known concentration of authentic pure samples in parallel tests.

\section{Examination of the effects of culture gases on Aspergillus niger}

A suspension of conidia of Aspergillus niger van Tieghem (Glasgow University Collection I) was made in $2 \%$ malt agar $(20 \mathrm{~g}$. Oxoid malt extract $+20 \mathrm{~g}$. Oxoid agar in I l. deionized water), at approximately $45^{\circ}$, poured immediately into a Petri dish and incubated for $18 \mathrm{~h}$. at $24^{\circ}$.

A series of I l. Roux bottles each containing $200 \mathrm{ml}$. of $2 \%$ malt agar were then each inoculated centrally with a $4 \times 2 \mathrm{~mm}$. disc cut from this suspension, the same 
suspension being used for all cultures in any one experiment. This inoculation method produced a single symmetrical colony in each vessel. All cultures were incubated at $24^{\circ}$ in a low light intensity. The effects of complete yeast culture gases were examined by joining Aspergillus niger cultures and yeast cultures by means of a glass T-piece passing through a rubber bung in the neck of each Roux bottle. Gas samples were taken by a syringe through a serum cap closing the lateral arm of the T-piece. Control cultures were paired with Roux bottles containing uninoculated $5 \%$ malt agar. The effects of identified organic components of the yeast-culture gas mixture were examined by placing samples of authentic pure compounds (purity verified by GLC) on filterpaper strips in the necks of the Roux bottles, and replacing the cotton-wool plug by a serum cap. The weight of each sample was that required to give an initial vapour pressure of the metabolite in the sealed vessel equal to the highest found at any stage during the incubation of yeast cultures with $A$. niger, or with cress seedlings. Untreated filter papers and serum caps were added to the control cultures at the same time.

Changes in $\mathrm{O}_{2}$ and in $\mathrm{CO}_{2}$ content during each experiment in the sealed assemblies were measured using a micro cross-section detector and column A $\left(\mathrm{O}_{2}\right)$ or column $\mathbf{B}$ $\left(\mathrm{CO}_{2}\right)$. Atmospheres with comparable $\mathrm{O}_{2}$ and $\mathrm{CO}_{2}$ concentrations to the final ones were made by partially evacuating freshly inoculated Roux bottles and replacing the air by $\mathrm{N}_{2}$ (for reduced $\mathrm{O}_{2}$ ) or by authentic $\mathrm{CO}_{2}$. Obviously by this method the $\mathrm{N}_{2}$ content was increased and the initial $\mathrm{CO}_{2}$ content was decreased in proportion to changes in the $\mathrm{O}_{2}$ content. It is thought that these changes can be disregarded at this stage, particularly as the reduction of $\mathrm{CO}_{2}$ concentration would be counteracted very quickly by respiration.

After 8 days incubation, linear growth of a colony was assessed by taking the mean of two diameters at right angles to each other, and any gross effects on sporulation were estimated by eye.

\section{Examination of the effects of culture gases on germination of cress seeds and on seedling growth}

Seeds of Lepidium sativum L. orient (cultivar Dobbies 'white curled' 1964, referred to as cress throughout this article) were sterilized by shaking in aqueous $\mathbf{H g C l}_{2}$ $(0.01 \%, w / v)$ for I min, followed by three changes of deionized water. Twenty sterilized seeds were placed in each of a series of $\mathrm{I} 1$. Roux bottles containing $200 \mathrm{ml} .0 .5 \%$ Oxoid agar in I l. deionized water. These were then paired with yeast cultures, control bottles, or received authentic single substances by the methods described above. All cultures were held at $22-26^{\circ}$ under continuous illumination from G.E.C. 'Warm White' fluorescent tubes.

After 5 days incubation a seed was recorded as germinated when it had produced a radicle as long as the diameter of the seed. Gross differences in the appearance of seedlings were noted by eye.

\section{RESULTS}

The only metabolites found in samples in sufficient concentration for identification were $\mathrm{CO}_{2}$, acetaldehyde, ethyl acetate, ethanol, $n$-propanol, isobutanol, and isopentanols. Oxygen concentrations below atmospheric were also noted. They were all found in samples both from pure yeast cultures and from the paired assemblies during biological activity tests. 
Figure I shows the chromatogram of a typical sample taken from a pure yeast culture after 2 days growth.

These are mostly common respiratory metabolites and their production is likely to be affected by many environmental factors. It was therefore deliberately decided to restrict the investigation to the study of their production and effects in the sealed assemblies used for the biological activity tests.

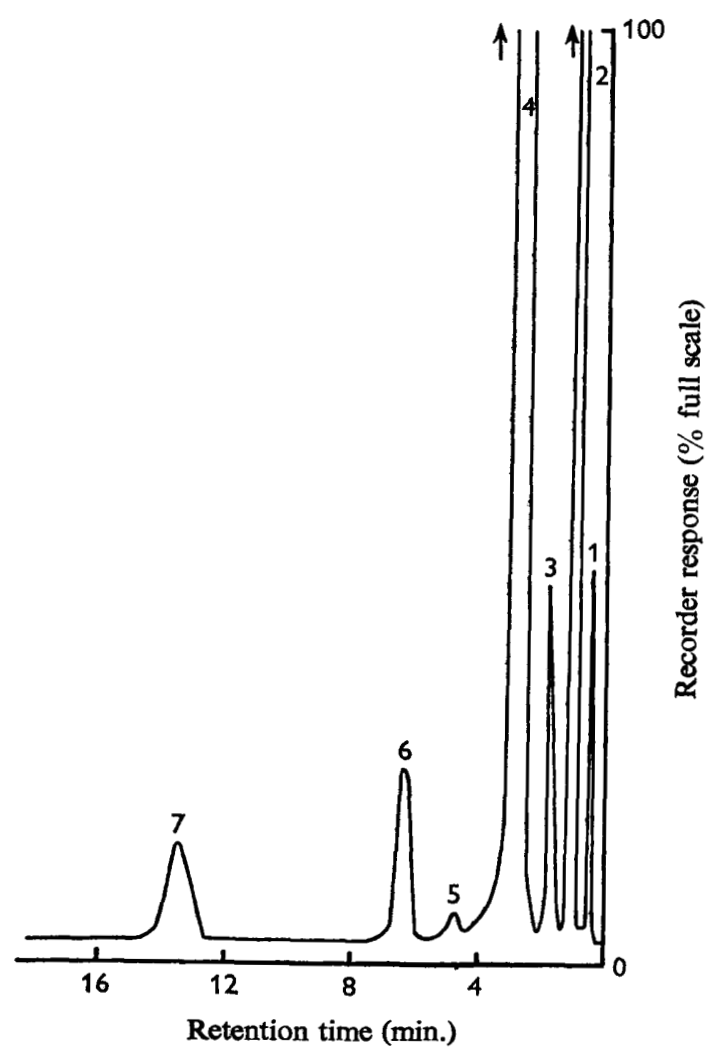

Fig. I. Typical chromatogram of Saccharomyces cerevisiae volatiles on column C (Table I) after 2 days growth. (1) Injection effect, (2) acetaldehyde, (3) ethyl acetate, (4) ethanol, (5) $n$-propanol, (6) isobutanol, (7) isopentanols.

Table 2. Volatile organic compounds identified in gases above Saccharomyces cerevisiae cultures

\section{Constituent}

Acetaldehyde

Ethyl acetate

Ethanol

n-Propanol

Isobutanol

2-Methyl-butan-I-ol

3-Methyl-butan-I-ol
Range of maximum concentration ( $\mu \mathrm{g} . / 1$. of air)

$$
\begin{gathered}
260-320 \\
13-15 \\
800-1000 \\
6-8 \\
12-16 \\
4-6 \\
8-10
\end{gathered}
$$


Table 2 shows the range of maximum concentrations of each metabolite measured in these tests. Figure 2 shows the changes in concentration of each measured in a representative test with Aspergillus niger. The changes followed this pattern consistently within the different ranges of concentration of each metabolite found in the three experiments in which this was followed.

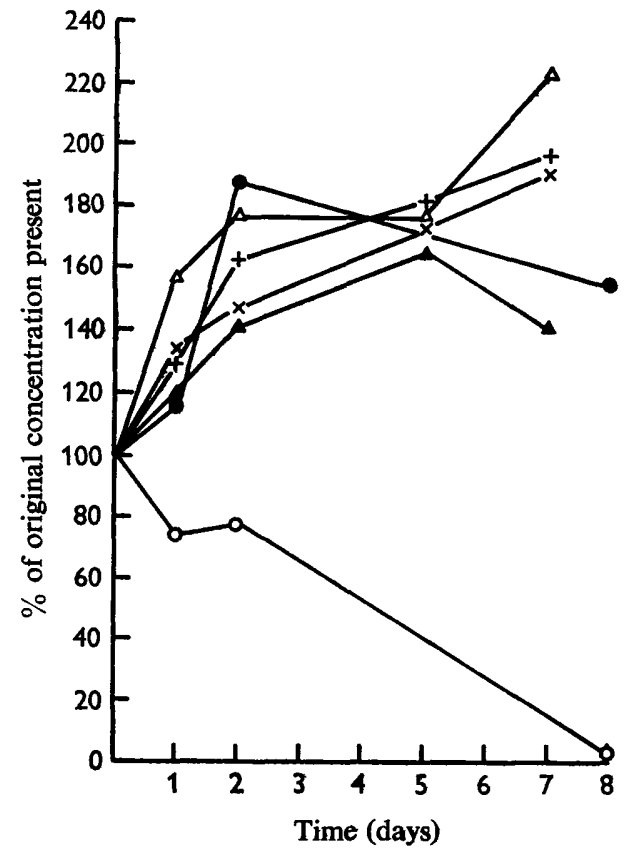

Fig. 2

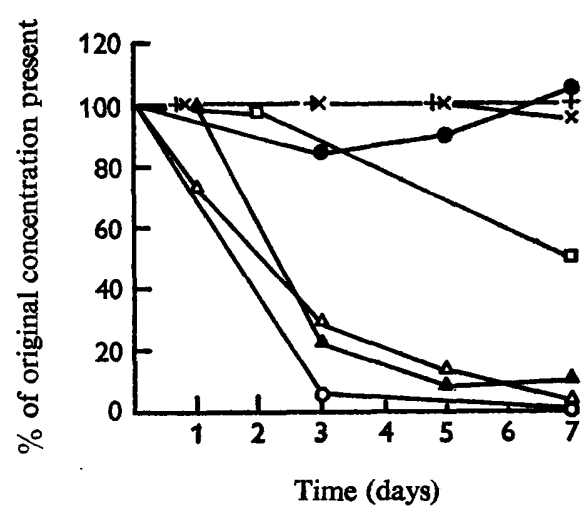

Fig. 3

Fig. 2. Changes in concentration of identified volatile metabolites in test assemblies of Aspergillus niger and yeast, expressed as \% of concentration of each, present immediately after the cultures were paired. Acetaldehyde, $\mathrm{C}-\mathrm{O}$; ethyl acetate $\triangle-\triangle$; ethanol, $-\infty$; $n$-propanol $\boldsymbol{\Delta}-\Delta$; isobutanol, $x-x$; 2-methyl-butan-I-ol+3-methylbutan-1-ol, +-+

Fig. 3. Changes in concentration of authentic pure compounds in tests with Aspergillis niger, expressed as percentage of concentration present at the start of the tests. The initial concentration of each was equal to the highest found at any stage in the other activity tests. Symbols on graph as in Fig. 2, with oxygen, $\square-\square$. Concentrations in $\mu$ g./l. of air: acetaldehyde, 320 ; ethyl acetate, 15 ; ethanol, 1000; $n$-propanol, 8 ; isobutanol, $16 ; 2$-methyl-butan- $1-01+3-$ methyl-butan-I-ol, I6.

The $\mathrm{O}_{2}$ content of the sealed assemblies containing yeast and Aspergillus niger decreased from approximately $13 \%(v / v)$ immediately after pairing to approximately $8 \%(\mathrm{v} / \mathrm{v})$, and the $\mathrm{CO}_{2}$ content increased from approximately $15 \%(\mathrm{v} / \mathrm{v})$ to approximately $25 \%(\mathrm{v} / \mathrm{v})$.

Figure 3 shows the initial concentration of authentic pure compounds in the synthetic gas/air mixtures, and the changes in this concentration during a representative test of the effect of the mixture on Aspergillus niger. Figure 4 compares the effects of the total culture gases and of the synthetic air/gas mixtures on the size of the diameter of $A$. niger colonies after 8 days growth. Each experiment involved the measurement and comparison of at least three cultures in each treatment with at least three controls and in each case at least three replicate experiments have given similar results. 
The diameters are reduced in the presence of complete culture gases and in the assemblies with an initial concentration of $320 \mu \mathrm{g}$. $/ 1$. acetaldehyde, or of $1000 \mu \mathrm{g} . / 1$. of ethanol, or of $25 \%(\mathrm{v} / \mathrm{v}) \mathrm{CO}_{2}$. These concentrations are the maximum found in any tests of yeast culture gases; since acetaldehyde concentration falls off rapidly while that of ethanol varies relatively slightly (Fig. 3), then it appears that both can contribute to the effects of complete culture gases; it seems likely that $\mathrm{CO}_{2}$ may also contribute to the effects of total culture gases in some conditions, although in these tests the concentration found only slightly exceeded the minimum inhibitory one (between $20 \%(\mathrm{v} / \mathrm{v})$ and $25 \%(\mathrm{v} / \mathrm{v})$ ). Sporulation, assessed by eye, was consistently

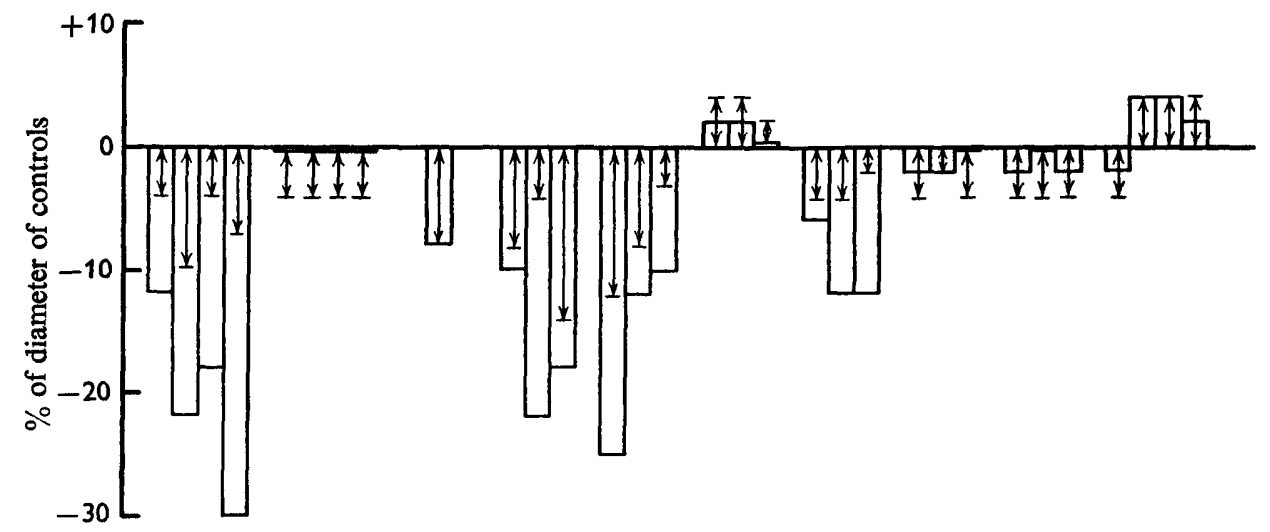

Fig. 4. Effects of yeast culture gases on diameters of colonies of Aspergillus niger. At least three replicates were used in each experiment. The vertical axis represents the mean diameters of test colonies expressed as a percentage of the mean diameter of the controls. $\leftrightarrow=$ least significant difference at $5 \%$ level. Each section on the horizontal axis represents a group of experiments using, from left to right, complete culture gases, $10 \% \mathrm{O}_{2}, 20 \% \mathrm{CO}_{2}$, $25 \% \mathrm{CO}_{2}$, acetaldehyde, ethyl acetate, ethanol, n-propanol, isobutanol, and 2-methylbutan-1-ol + 3-methyl-butan-I-ol.

reduced by about half by the presence of complete culture gases, or of $25 \%(\mathrm{v} / \mathrm{v})$ initial $\mathrm{CO}_{2}$ concentration, and by about one-third in the presence of $20 \%$ initial $\mathrm{CO}_{2}$ concentration. It seems likely therefore that $\mathrm{CO}_{2}$ may contribute to the effects. No other identified compounds have been shown to have any significant biological effect on Aspergillus niger in the concentrations tested. The possibility that ethyl acetate and/or $n$-propanol can contribute to the effects of the total culture gases has not been eliminated, however, as their concentrations fall off rapidly in the tests of authentic pure compounds. Our reasons for deliberately deciding not to investigate this possibility further are given in the discussion below.

The $\mathrm{O}_{2}$ content of the sealed assemblies containing yeast and cress seeds decreased from approximately $17 \%(\mathrm{v} / \mathrm{v})$ immediately after pairing to approximately $10 \%(\mathrm{v} / \mathrm{v})$, and; the $\mathrm{CO}_{2}$ content increased from approximately $7 \%(\mathrm{v} / \mathrm{v})$ to approximately $20 \%(\mathrm{v} / \mathrm{v})$. The range of concentrations of other identified metabolites found in any test is shown in Table 2, and the changes in concentration measured during a representative test are shown in Fig. 5. Figure 6 shows the initial concentration of pure compound in the synthetic gas-air mixture and the changes in this concentration during a representative test. Figure 7 shows the effects of total culture gases and of pure compounds on seed 
germination. Each experiment involved the measurement and comparison of at least four cultures in each treatment with at least four controls, and at least three replicate experiments have given similar results.

Germination is significantly reduced in the presence of complete culture gases, in assemblies with $10 \%(\mathrm{v} / \mathrm{v})$ initial $\mathrm{O}_{2}$, and in those with an initial concentration of $1000 \mu \mathrm{g} . / 1$. ethanol or of $10 \mu \mathrm{g} . / 1$. 3-methyl-butan-I-ol. Since this is the maximum

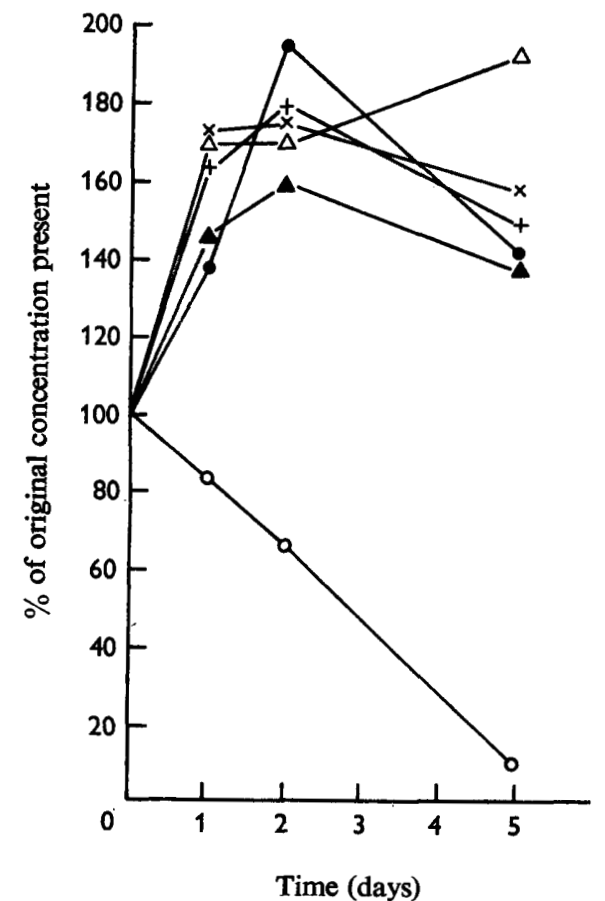

Fig. 5

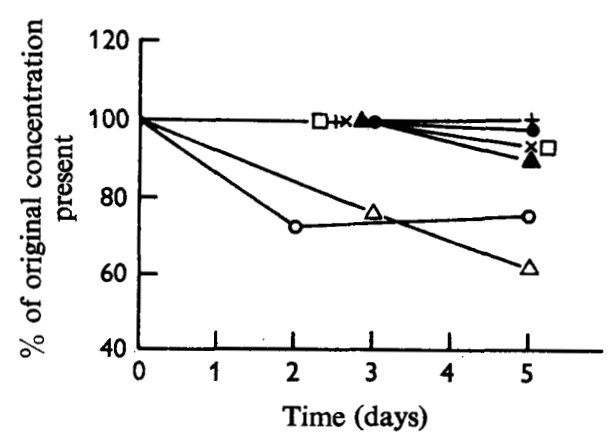

Fig. 6

Fig. 5. Changes in concentration of identified volatile metabolites in test assemblies of yeast and cress seeds, expressed as percentage of concentration present at start of test. Symbols on graph as in Fig. 2.

Fig. 6. Changes in concentration of authentic pure compounds in tests with cress seeds, expressed as percentage of concentration present at the start of the test. The initial concentration of each was equal to the highest found at any time in other activity tests. Symbols on graph as in Fig. 2. Concentrations in $\mu \mathrm{g} . / 1$. of air: acetaldehyde, 320; ethyl acetate, 15; ethanol, 1000; $n$-propanol, 8; isobutanol, 12; 2-methyl-butan-1-ol, 6; 3-methyl-butan-I-ol, Io.

concentration found in any tests of yeast culture gases, and since the ethanol and 2-methyl-butan-I-ol concentrations vary only slightly (Figure 6), then it appears that both these can contribute to the effects of the complete gases. The significant reduction of germination in experiments with acetaldehyde at $500 \mu \mathrm{g} . / 1$. of air (i.e. $\times \mathrm{I} \cdot 5$ the highest concentration above yeast cultures) and in those using isobutanol at $30 \mu \mathrm{g} . / \mathrm{l}$. of air (i.e. twice the yeast culture concentration) suggest that these might have an effect in some conditions.

The evidence that the concentration of acetaldehyde fell off rapidly during all these tests suggests, however, that it does not contribute to the effect in these conditions. 
The possibility that ethyl acetate can contribute to the effect of the total culture gases has not been entirely eliminated, as its concentration falls off by $40 \%$ during the test of the authentic pure compound. Our reasons for deliberately deciding not to investigate this further are given in the discussion below.

The few seedlings which grew in cultures with complete culture gases or with ethanol, and those which grew with $\mathrm{CO}_{2}(20 \%, v / v)$ were severely stunted and chlorotic; in most of these cultures growth was restricted to formation of a radicle a few millimetres long. They were less stunted and less chlorotic in cultures with acetaldehyde. The few seedlings which grew in the presence of 3-methyl-butan-I-ol were slightly stunted but similar in colour to those in the controls and to those in cultures with ethyl acetate, $n$-propanol and isobutanol.

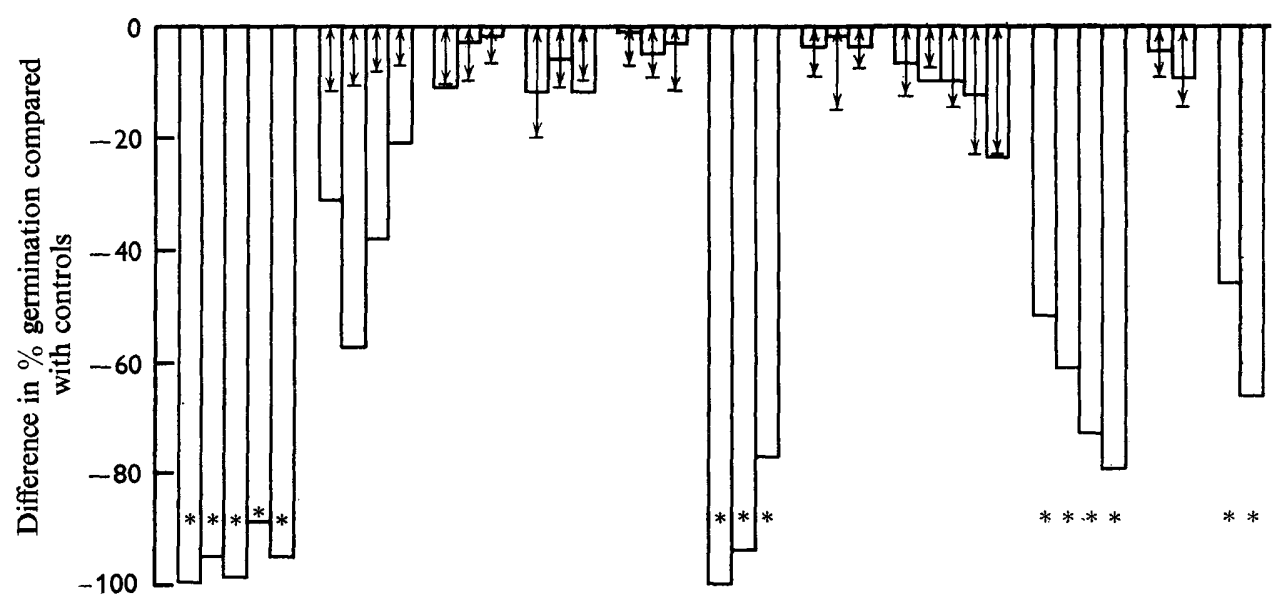

Fig. 7. Effects of yeast culture gases on germination of cress seeds. At least three replicates were used in each experiment. The vertical axis represents the mean germination of test cultures expressed as a percentage of the mean germination of the controls. $\leftrightarrow=$ least significant difference at $5 \%$ level. ${ }^{*}=$ Differences clearly significant by empirical observation. Each section on the horizontal axis represents a group of experiments using, from left to right, complete culture gases, $10 \% \mathrm{O}_{2}, 20 \% \mathrm{CO}_{2}$, acetaldehyde, ethyl acetate, ethanol, $n$-propanol, isobutanol, 2-methyl-butan-I-ol + 3-methyl-butan-I-ol, 2-methyl-butan-I-ol, and 3-methylbutan-I-ol.

\section{DISCUSSION}

No unusual metabolites have been found in the yeast culture gases; their biological effects can be due to the production of a number of common respiratory metabolites which may be effective singly or as parts of a mixture. The results contrast with those of the study of Fomes annosus (Glen, Hutchinson \& McCorkindale, 1966), which showed that the biological activity of this fungus is due to the production of a single unusual metabolite (hexa-I,3,5-triyne); the effects on hyphal growth of $A$. niger extend the work of Robinson et al. (1966, 1968), who report that acetaldehyde and ethanol had no such effects on the species which they examined in these conditions. Only a limited comparison can be made with the results of Norrman (1968), owing to differences in concentrations and conditions tested. The differences in response to ethanol may be related to the fact that the maximum concentrations he tested were less than one-fifth of those found in the yeast/Aspergillus assemblies and used in our tests of 
authentic pure ethanol. In his tests with Pestalotia rhododendri acetaldehyde, npropanol, and isobutanol stimulated sporulation, isobutanol and $n$-propanol inhibited linear growth, all at the concentrations similar to those at which they showed no appreciable effect on Aspergillus niger colonies in our tests of authentic pure compounds. He found that $0 . \mathrm{I} \%$ 'iso-amyl alcohol' inhibited sporulation; it is not, however, possible to compare this result with our tests, as he does not specify whether this is a mixture of 2-methyl-butan-I-ol and 3-methyl-butan-I-ol or pure 3-methylbutan-I-ol. Neither $\boldsymbol{P}$. rhododendri nor $\boldsymbol{A}$. niger were apparently affected by similar concentrations of ethyl acetate.

The composition of the gas mixtures of these common metabolites from yeast will vary greatly in different cultural conditions and hence any detailed analysis of one condition would have very limited general biological application. At the present time it does not seem justifiable to continue the analysis of such obviously complex possible interactions and of the possible patterns in the cumulative effects of mixtures. The report is therefore published as an illustration of a complex interaction of a mixture of common respiratory metabolites which may have obvious ecological importance in some conditions-for example, in the ecology of soil micro-organisms.

The authors are very grateful to the Science Research Council for a grant which supported this work, to Professor Brian and Professor Raphael for facilities in their respective departments, to Dr C. J. W. Brooks for advice on gas chromatography, and to Mrs J. M. Mackintosh and the Technical Staff of the laboratory for much assistance.

\section{REFERENCES}

Dick, C. M. \& Hutchinson, S. A. (1966). Biological effects of volatile fungal metabolites. Nature, Lond. 2II, 868.

Glen, A. T., Hutchinson, S. A. \& McCorkindale, N. J. (1966). Hexa-I,3,5-triyne-a metabolite of Fomes annosus. Tetrahedron Lett. p. 4223.

HoFf, J. E. \& FerT, E. D. (1964). New technique for functional group analysis in gas chromatography. Analyt. Chem. 36, 1002.

Hutchinson, S. A. (1967). Some effects of volatile fungal metabolites on the gametophytes of Pteridium aquilinum. Trans. Br. mycol. Soc. 50, 333.

Lösel, D. M. (1964). The stimulation of spore germination in Agaricus bisporus by living mycelium. Ann. Bot. N.S. 28, 54I.

NORRMAN, J. (I968). Morphogenetic effects of some volatile organic compounds of Pestalotia rhododendri. Arch. Mikrobiol. 6r, 128.

Robinson, P. M. \& PARK, D. (1966). Volatile inhibitors of spore germination produced by fungi Trans. Br. mycol. Soc. 49, 639.

Robinson, P. M., PARK, D. \& Garret, M. K. (1968). Sporostatic products of fungi. Trans. Br. mycol. Soc. 5r, II3. 